\title{
Alterações hemodinâmicas identificadas durante banho no leito do paciente crítico: revisão integrativa
}

\author{
Hemodynamic changes identified during bathing in the critical patient's bed: integrative review \\ Cambios hemodinámicos identificados durante el baño en la cama del paciente crítico: revisión \\ integrativa
}

Recebido: 11/10/2021 | Revisado: 16/10/2021 | Aceito: 23/10/2021 | Publicado: 24/10/2021

Layala de Souza Goulart

ORCID: https://orcid.org/0000-0001-6575-1831 Hospital Regional de Mato Grosso do Sul, Brasil E-mail: layalasouza@gmail.com

Nivea Lorena Torres

ORCID: https://orcid.org/0000-0003-3715-8840 Hospital Regional de Mato Grosso do Sul, Brasil E-mail: nivealorenatorres@gmail.com

Rodrigo Guimarães dos Santos Almeida ORCID: https://orcid.org/0000-0002-4984-3928 Universidade Federal de Mato Grosso do Sul, Brasil E-mail: rgclaretiano@gmail.com

Nayara Albina Freitas Souza

ORCID: https://orcid.org/0000-0002-9125-1870 Hospital Regional de Mato Grosso do Sul, Brasil E-mail: nay_afs@hotmail.com

\begin{abstract}
Resumo
Objetivo: analisar as evidencias cientificas a respeito das alterações hemodinâmicas apresentadas pelo paciente crítico durante banho no leito. Métodos: Trata-se de uma revisão integrativa da literatura, após utilizar a estratégia PICO, construímos a seguinte pergunta norteadora "Quais as possíveis alterações hemodinâmicas apresentadas pelo paciente crítico relacionadas ao banho no leito?". Com a leitura e aplicação dos critérios de seleção foram utilizados cinco artigos. Resultados: os artigos puderam ser agrupados em duas categorias - Alterações hemodinâmicas relacionadas ao tipo de banho e as principais alterações hemodinâmicas encontradas durante o banho, independente, do tipo de banho. Os artigos revisados obtiveram resultados significativos quanto as variáveis hemodinâmicas: temperatura corporal, frequência respiratória (FR) e cardíaca (FC), Pressão Arterial (PA), índice cardíaco, Pressão Venosa Central (PVC) e volume sistólico. Conclusão: Evidenciou-se a necessidade de realizar investigações mais minuciosas para melhor esclarecimento dos impactos do banho no leito ao paciente crítico, para uma assistência de enfermagem com embasamento em evidências cientificas e segura.
\end{abstract}

Palavras-chave: Enfermagem; Cuidados críticos; Banho no leito.

\begin{abstract}
Objective: to analyze the scientific evidence regarding hemodynamic changes presented by the critically ill patient during a bed bath. Methods: This is an integrative literature review, after using the PICO strategy, we built the following guiding question "What are the possible hemodynamic changes presented by critically ill patients related to bed baths?". With the reading and application of the selection criteria, five articles were used. Results: the articles could be grouped into two categories - Hemodynamic changes related to the type of bath and the main hemodynamic changes found during the bath, regardless of the type of bath. The reviewed articles obtained significant results regarding the hemodynamic variables: body temperature, respiratory (RR) and heart (HR), Blood Pressure (BP), cardiac index, Central Venous Pressure (CVP) and systolic volume. Conclusion: The need for more detailed investigations to better clarify the impacts of bed baths for critically ill patients was evidenced, for nursing care based on scientific and safe evidence.
\end{abstract}

Keywords: Nursing; Critical care; Bed bath.

\section{Resumen}

Objetivo: analizar la evidencia científica sobre los cambios hemodinámicos que presenta el paciente crítico durante un baño en cama. Métodos: Se trata de una revisión integradora de la literatura, luego de utilizar la estrategia PICO, se construyó la siguiente pregunta orientadora "¿Cuáles son los posibles cambios hemodinámicos que presentan los pacientes críticamente enfermos relacionados con los baños en cama?". Con la lectura y aplicación de los criterios de 
selección se utilizaron cinco artículos. Resultados: los artículos se pudieron agrupar en dos categorías - Cambios hemodinámicos relacionados con el tipo de baño y los principales cambios hemodinámicos encontrados durante el baño, independientemente del tipo de baño. Los artículos revisados obtuvieron resultados significativos en las variables hemodinámicas: temperatura corporal, respiratoria (RR) y cardíaca (FC), presión arterial (PA), índice cardíaco, presión venosa central (PVC) y volumen sistólico. Conclusión: Se evidenció la necesidad de investigaciones más detalladas para esclarecer mejor los impactos de los baños en cama para pacientes críticos, para el cuidado de enfermería basado en evidencia científica y segura.

Palabras clave: Enfermería; Cuidado crítico; Baño en la cama.

\section{Introdução}

O banho é um cuidado de higiene realizado pela enfermagem que pode ser realizado no leito aos pacientes com restrições, de forma completa ou parcial. No banho completo é realizada higiene de todos os segmentos corporais e no parcial é higienizada apenas as partes do corpo que causariam muito desconforto se não lavadas, como face, axilas, região perineal e mãos (Potter \& Perry, 2013).

Outra modalidade de banho no leito é o a seco desenvolvido para atender aos pacientes com predisposição à pele seca e a risco de infecção, devido aos utensílios utilizados para o banho (bacias/baldes de banho). Realizado com a utilização de compressas descartáveis umedecidas com solução emoliente, que também contribui para reduzir o tempo de exposição do paciente (Potter \& Perry, 2013; Toledo, et al., 2021).

As Unidades de Terapia Intensiva (UTI), destinam-se ao atendimento de pacientes graves e instáveis. O ambiente das UTI's são considerados de alta complexidade devido à característica invasiva dos procedimentos realizados, que envolvem materiais específicos, profissionais qualificados e tecnologias especializadas para a monitorização, diagnóstico e terapêutica necessários ao atendimento desses pacientes (Backes; Erdmann \& Buscher, 2015; Costa, et al., 2018).

São caracterizados como pacientes graves aqueles que apresentam comprometimento de um ou mais sistemas dos sistemas fisiológicos, com perda da autorregulação, com necessidade de assistência contínua (Brasil, 2010).

A monitoração hemodinâmica do paciente critico tem por objetivo verificar de maneira direta ou indireta, através de diversas variáveis hemodinâmicas, a entrega adequada de oxigênio tecidual e a perfusão de órgãos, essencial para compreensão do estado e manejo clínico, com finalidade de obter melhores resultados na sobrevida do paciente (Viana, Whitaker \& Zanei, 2020).

As medidas de monitorização são reflexo indireto do débito cardíaco, por meio de variáveis de fácil obtenção como temperatura, frequência cardíaca e respiratória, pressão arterial sistêmica, saturação arterial periférica de oxigênio e diurese. A literatura aponta mensurações estáticas e dinâmicas para avaliação do paciente crítico e seu estado volumétrico (Viana, Whitaker \& Zanei, 2020).

Neste contexto o banho no leito do paciente crítico necessita de planejamento e organização, visto que toda a tecnologia que oferece suporte à vida do paciente e auxilia no seu monitoramento são fatores que dificultam a mobilidade e o acesso ao paciente, bem como o cuidado com as alterações hemodinâmicas (Flores, 2016).

O trabalho da enfermagem na UTI é complexo e, como tal, requer ponderar variáveis necessárias para o desenvolvimento do cuidado, como a dinâmica entre os profissionais, a condição crítica dos pacientes e a utilização de inúmeras tecnologias, o que demanda da enfermagem conhecimentos de ordens diversas, potencializando a assistência prestada e maximizando processos efetivos de trabalho e cuidado (Massaroli, et al., 2015).

Apesar de ser um procedimento comum dentre os procedimentos de enfermagem, muitas vezes é realizado sem raciocínio crítico, pela incorporação na rotina de cuidados de enfermagem. Assim sendo, faz-se necessária uma avaliação antes da realização do banho no leito, o que inclui os parâmetros hemodinâmicos (Flores, 2016).

O momento do banho também se faz uma oportunidade para estimulação da circulação, mobilidade, manutenção de 
conforto e higiene do paciente, assim como avaliação cutânea e as condições dos dispositivos. Em que se faz primordial a checagem das fixações e estabilidade desses dispositivos antes de iniciar a manipulação. Sendo assim uma oportunidade do enfermeiro de avaliar o paciente, de forma detalhada, para realização do processo de enfermagem (Viana, 2011).

Diante de tal problemática, pretende-se com esta revisão responder a questão "Quais as possíveis alterações hemodinâmicas apresentadas pelo paciente crítico relacionadas ao banho no leito?"

Desta forma, o objetivo desta revisão é analisar as evidencias cientificas a respeito das alterações hemodinâmicas apresentadas pelo paciente crítico durante banho no leito.

\section{Metodologia}

Trata-se de uma revisão integrativa da literatura, que é um método de pesquisa aplicável à prática clínica para embasamento da Prática Baseada em Evidência (PBE). Tem a finalidade de reunir e sintetizar resultados de pesquisas sobre um tema, de maneira sistemática e ordenada, contribuindo para o aprofundamento do conhecimento do tema estudado. E, assim fornecendo suporte para a tomada decisão na prática clínica, além de evidenciar as lacunas de conhecimento sobre determinado tema (Mendes, Silveira \& Galvão, 2008; Costa, et al., 2018).

Para a elaboração desta revisão integrativa, foram realizadas as seguintes etapas: determinação do objetivo específico, formulação do questionamento a ser respondido, busca para identificar e coletar o máximo de pesquisas primárias relevantes, dentro dos critérios de inclusão e exclusão estabelecidos.

A pergunta norteadora da pesquisa foi elaborada através da estratégia PICO, que representa o acrônimo Paciente, Intervenção, Comparação e Outcomes (desfecho), que para esta revisão foi utilizada conforme a Tabela 1 (Santos, Pimenta \& Nobre, 2007).

Tabela 1. Aplicação da estratégia PICO.

\begin{tabular}{|l|l|}
\hline Estratégia PICO \\
\hline Paciente & Paciente crítico \\
\hline Intervenção & Banho no leito \\
\hline Comparação & Alterações hemodinâmicas \\
\hline Outcomes & \\
\hline
\end{tabular}

Fonte: Elaboração própria.

Os termos de busca selecionados, segundo descritor em saúde, foram banhos, cuidados críticos, enfermagem de cuidados críticos e monitorização hemodinâmica, e utilizando-se dos operadores boleanos "AND" e "OR".

As bases de dados utilizadas para busca foram Biblioteca Virtual em Saúde (BVS) que inclui as bases de dados LILACS, MEDLINE, BDENF e SCIELO, e PubMed, no mês de agosto de 2021.

Foram incluídos estudos originais desenvolvidos com pacientes: maiores de 18 anos; criticamente enfermos; submetidos a banho no leito; que apresente as alterações hemodinâmicas mensuradas ou observadas; e disponíveis para leitura de forma completa nos idiomas português e inglês.

Foram excluídos estudos desenvolvidos com: menores de 18 anos; paciente internados em unidades de internação; submetidos a outros métodos de banho, que não seja no leito; estudos de revisão integrativa, dissertações, monografias e teses; que não mensure ou observe as alterações hemodinâmicas decorrentes do banho no leito; e indisponíveis para leitura na integra.

Após a consulta nas bases de dados que totalizou 122 artigos, e a aplicação dos critérios inclusão e exclusão, foram 
realizadas as seguintes etapas (Figura 1). Após leitura do título foram selecionados para leitura do resumo 15 artigos, e cinco selecionados para leitura na integra e incluídos na revisão.

Figura 1 - Processo de seleção dos artigos.

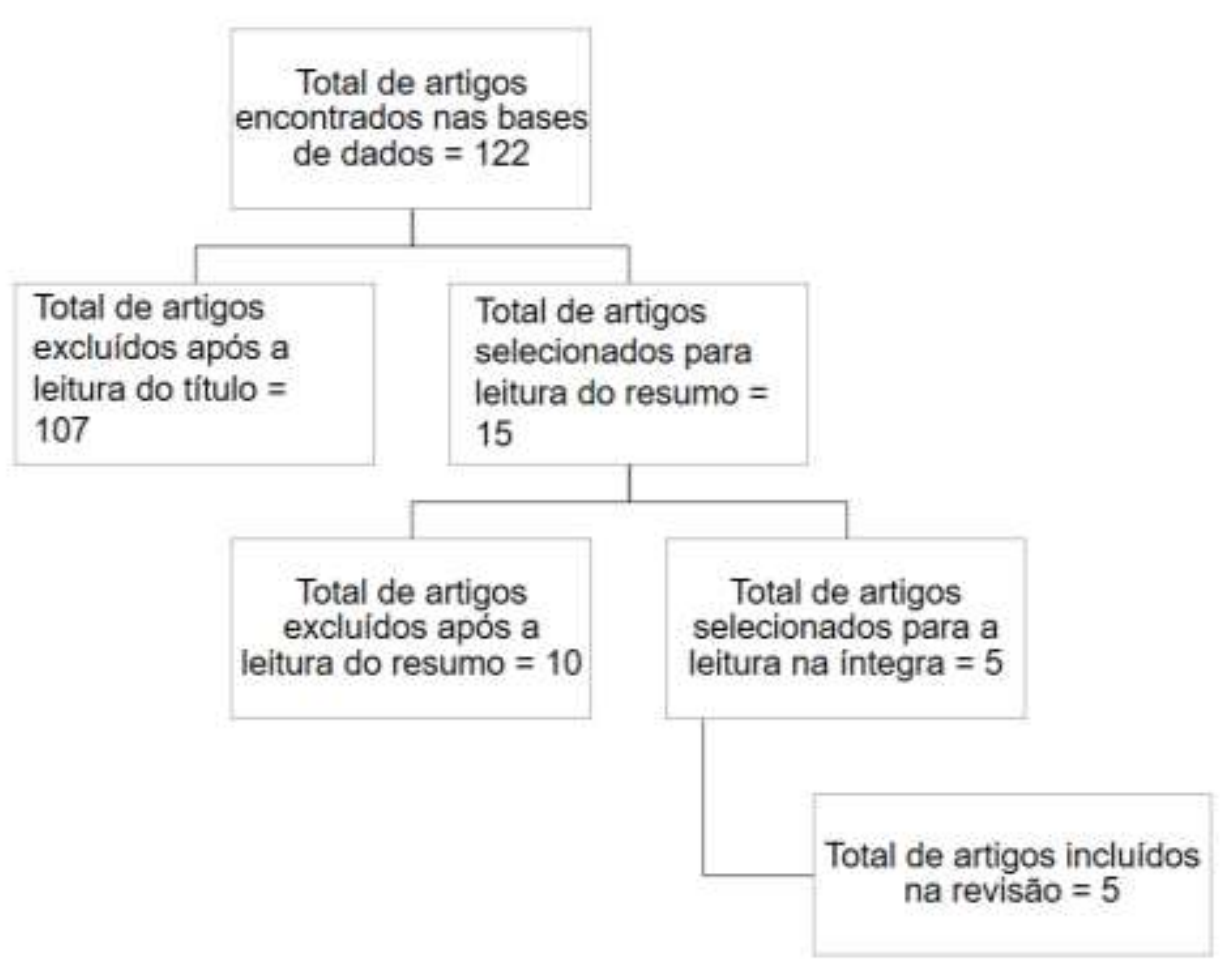

Fonte: Elaboração própria.

\section{Resultados e Discussão}

Nesta revisão, foram utilizados cinco artigos publicados (Quadro 1), a partir de 2012, os resultados obtidos foram agrupados em duas categorias: alterações hemodinâmicas relacionadas ao tipo de banho e as principais alterações hemodinâmicas encontradas durante o banho, independente, do tipo de banho. Os artigos foram classificados dentro de sete níveis de evidencias, conforme Galvão (2006), apresentando níveis de evidência dois e três.

Quadro 1 - Síntese dos artigos que relacionam as alterações hemodinâmicas durante o banho no leito do paciente crítico.

Campo Grande, Mato Grosso do Sul, Brasil. 2021.

\begin{tabular}{|c|c|c|c|}
\hline $\begin{array}{l}\text { Autor/ano de } \\
\text { publicação }\end{array}$ & Objetivo e delineamento & $\begin{array}{c}\text { Nível de } \\
\text { evidência }\end{array}$ & Resultado \\
\hline $\begin{array}{l}\text { Madrid, S. Q.; } \\
\text { Lopez, C. C.; } \\
\text { Otalvaro, A. F. T.; } \\
\text { Padilla, L. M. R., } \\
2012\end{array}$ & $\begin{array}{l}\text { Avaliar as alterações hemodinâmicas } \\
\text { que se apresentam durante a realização } \\
\text { do banho no leito em pacientes críticos } \\
\text { no pós-operatório de cirurgia cardíaca. } \\
\text { Estudo quase experimental }(\mathrm{n}=36)\end{array}$ & Nível 3 & $\begin{array}{l}\text { A maioria recebeu banho de esponja }(94,3 \%) \text {. O diagnóstico } \\
\text { pós-operatório mais frequente foi revascularização } \\
\text { coronariana }(70 \%) \text {. No decorrer do tempo de avaliação do } \\
\text { paciente foram observadas alterações nos valores de pressão } \\
\text { arterial sistólica e frequência respiratória }(\mathrm{p}=0,017 \text { e } \mathrm{p} \\
<0,0001 \text {, respectivamente). Também observou-se aumento } \\
\text { da pressão arterial sistólica durante o banho }(\mathrm{p}=0,010) \text { e } \\
\text { aumento da frequência respiratória após o banho (p = } \\
0,008) \text {. }\end{array}$ \\
\hline $\begin{array}{l}\text { Ramos, J. M. F.; } \\
\text { Consuegra, R. V. G.; }\end{array}$ & $\begin{array}{l}\text { Avaliar os efeitos de dois tipos de } \\
\text { banho de leito: secos e tradicionais, em }\end{array}$ & Nível 3 & $\begin{array}{l}\text { Não foram encontradas alterações nos parâmetros de } \\
\text { pressão arterial } \mathrm{e} \text { frequência respiratória durante a }\end{array}$ \\
\hline
\end{tabular}




\begin{tabular}{|c|c|c|c|}
\hline $\begin{array}{c}\text { Urrego, K. A. G., } \\
2016\end{array}$ & 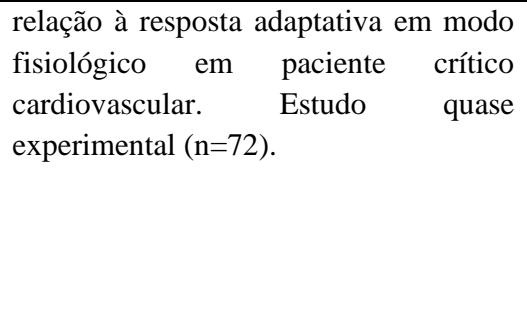 & & $\begin{array}{l}\text { realização do banho no leito a seco. Entretanto, em } 44,4 \% \\
\text { dos pacientes submetidos ao banho no leito tradicional } \\
\text { houve necessidade de pelo menos um cuidado adicional } \\
\text { para estabilização hemodinâmica, tais como uso de } \\
\text { medicações vasoativas ou inotrópicas }(16,7 \%) \text { e } \\
\text { modificações de parâmetros ou dispositivos ventilatórios } \\
(11,1 \%) \text {. No banho a seco } 91,7 \% \text { dos pacientes não } \\
\text { necessitaram de nenhum cuidado adicional. }\end{array}$ \\
\hline $\begin{array}{l}\text { Toledo, L. V.; } \\
\text { Salgado, P. O.; } \\
\text { Souza, C. C.; Brinati, } \\
\text { L. M.; Januario, C. } \\
\text { F.; Ercole, F. F., } \\
2020\end{array}$ & $\begin{array}{l}\text { Comparar o tempo de execução do } \\
\text { banho no leito a seco e do tradicional, e } \\
\text { os seus efeitos sobre a saturação } \\
\text { transcutânea de oxigênio arterial e a } \\
\text { frequência respiratória em pacientes } \\
\text { críticos adultos. Ensaio clínico } \\
\text { randomizado }(n=15)\end{array}$ & 2 & $\begin{array}{l}\text { A maioria dos pacientes era do sexo masculino, brancos, } \\
\text { com média de idade de } 69,7 \text { anos. O banho a seco foi mais } \\
\text { rápido, que o tradicional }(\mathrm{p}<0,001 \text { ). Não houve diferença } \\
\text { significativa entre as médias de saturação dos pacientes } \\
\text { entre os banhos ( } \mathrm{p}=0,381 \text { ), sendo } 94,7 \% \text { no banho a seco e } \\
95,2 \% \text { no tradicional. Durante o banho tradicional, a média } \\
\text { da frequência respiratória dos pacientes foi maior e } \\
\text { estatisticamente diferente ( }<<0,001 \text { ) do valor obtido no } \\
\text { banho a seco. }\end{array}$ \\
\hline $\begin{array}{l}\text { Reis, F. F.; Silva, L. } \\
\text { S.; Spitz, V. M.; } \\
\text { Silva, M. E. S.; } \\
\text { Oliveira, A. P.; } \\
\text { Lima, D. V. M., } \\
2021\end{array}$ & $\begin{array}{l}\text { Analisar o impacto oxihemodinâmico } \\
\text { do banho no leito sem controle da } \\
\text { temperatura da água realizado em até } \\
20 \text { minutos em pacientes com infarto } \\
\text { agudo do miocárdio. Ensaio clínico não } \\
\text { controlado (n=23) }\end{array}$ & & $\begin{array}{l}\text { Maioria do sexo masculino e idade média } 68 \text { anos. A maior } \\
\text { incidência foi de Infarto Agudo do Miocárdio com Supra } \\
\text { desnivelamento do segmento ST. Houve pouca variação da } \\
\text { frequência cardíaca e da pressão arterial antes, durante e } \\
\text { após o banho. O índice cardíaco e volume sistólico } \\
\text { apresentaram variações significativas, sem provocar } \\
\text { instabilidade clínica. }\end{array}$ \\
\hline $\begin{array}{l}\text { Toledo, L. V.; } \\
\text { Santos, B. X.; } \\
\text { Salgado, P. O.; } \\
\text { Souza, L. M.; } \\
\text { Brinati, L. M.; } \\
\text { Januário, C. F.; } \\
\text { Ercole, F. F., } 2021\end{array}$ & $\begin{array}{l}\text { Comparar os valores de temperatura } \\
\text { corporal timpânica e axilar dos } \\
\text { pacientes críticos antes e depois da } \\
\text { realização do banho no leito tradicional } \\
\text { e a seco. Ensaio clínico randomizado } \\
(\mathrm{n}=50) \text {. }\end{array}$ & Nível 2 & $\begin{array}{l}\text { Predominaram os pacientes idosos e do sexo masculino. } \\
\text { Não houve diferença significativa entre as medianas de } \\
\text { temperatura timpânica aferidas durante o banho no leito } \\
\text { tradicional ( } \mathrm{p}=0,707 \text { ) e a seco ( } \mathrm{p}=0,101 \text { ). As médias da } \\
\text { temperatura axilar reduziram ao final dos banhos ( } \mathrm{p}=0,001 \text { ), } \\
\text { sendo } 36,12{ }^{\circ} \mathrm{C} \text { no banho tradicional e } 35,92^{\circ} \mathrm{C} \text { no banho a } \\
\text { seco. Independente do método utilizado, o banho no leito } \\
\text { foi capaz de provocar alterações no equilíbrio térmico dos } \\
\text { pacientes críticos. }\end{array}$ \\
\hline
\end{tabular}

Fonte: Elaboração própria.

Os artigos revisados obtiveram resultados significativos quanto as variáveis hemodinâmicas: temperatura corporal, frequência respiratória (FR) e cardíaca (FC), Pressão Arterial (PA), índice cardíaco, PVC e VS. Também houve a avaliação do tipo de banho e tempo dispendido para realização em três artigos (60\%), que evidenciou alterações menos significativas no banho a seco, ou seja, quando há menor tempo de exposição e manipulação do paciente.

\section{Alterações hemodinâmicas relacionadas ao tipo de banho}

Em estudo de Ramos, et al. (2016) observou alterações de frequência cardíaca e pressão venosa central durante a realização do banho, além de hipertensão e taquipneia associada a realização do banho tradicional, eventos não observados no banho a seco.

Houve significância estatística na variação da temperatura axilar dos pacientes após o banho no leito, apresentando valores menores que os iniciais, em ambos os métodos de banho (Toledo, et al., 2021).

Foi evidenciada a média de tempo do banho no leito tradicional, que foi de 30 minutos, e do banho a seco, de 20 minutos. Em relação a frequência respiratória foi maior e estatisticamente diferente no banho no leito tradicional quando comparado ao banho a seco. No entanto, não foi encontrada diferença estatística nas mensurações de saturação de oxigênio nos diferentes tipos de banhos (Toledo, et al., 2020). 


\section{As principais alterações hemodinâmicas encontradas durante o banho, independente, do tipo de banho}

Quando avaliados os parâmetros hemodinâmicos antes, durante e após o banho, o que houve significância estatística foram as alterações de Pressão Arterial Sistólica (PAS) durante o banho e FR após o banho. O tempo médio na execução do banho quando realizado por apenas um profissional de enfermagem foi de 30-40 minutos e por dois profissionais de 20-25 minutos, expondo o paciente a maior tempo de manipulação quando realizado por apenas um profissional (Madrid, et al., 2012).

Quando avaliados os pacientes com infarto agudo do miocárdio durante o banho no leito foram observadas queda do volume sistólico, do tempo de ejeção ventricular esquerdo, do trabalho cardíaco esquerdo e índice de oferta de oxigênio, porém sem repercussão com instabilidade para os pacientes. No caso dos pacientes com infarto sem supradesnivelamento do seguimento ST apresentaram elevação das variáveis PAS, PAM, trabalho cardíaco esquerdo e índice de oferta de oxigênio com retorno dos valores em repouso inicial após cinco minutos do término do banho (Reis, et al., 2021).

As atividades de assistência de enfermagem devem ser desenvolvidas de forma a minimizar os riscos relacionados a prestação de assistência, que deve ser aplicada na realização do banho no leito.

Uma revisão sistemática recentemente apresentou que pacientes graves aparentemente se beneficiam de algumas ações para realização do banho no leito, como sempre evitar o banho em menos de 4 horas após a cirurgia cardíaca; tentar ser o mais breve possível no posicionamento do paciente em decúbito lateral e concluir o banho dentro de 20 minutos. O que corrobora com os achados em que houve menos repercussões hemodinâmicas no banho a seco com menor tempo de execução e exposição do paciente. Assim, o que faz necessário um planejamento para execução deste cuidado quanto aos materiais, organização, tempo de execução e mudanças de posicionamento (Marins \& Cruz, 2016).

O tempo gasto para realização do banho corrobora com achado de repercussão hemodinâmica por Toledo, et al. (2020) e Ramos, et al. (2016), onde a FR, FC e pressão venosa central foi maior e com significância estatística no banho tradicional, em que se leva mais tempo de execução e com maior exposição e manipulação do paciente, quando comparado ao banho a seco.

Quanto ao tempo de execução do banho e exposição do paciente, estudo descreveu que o empenho físico é um fator de risco para segurança dos profissionais devido ao desgaste físico e está relacionado com a carga de trabalho da equipe de enfermagem. Diante disso, é necessário ter um dimensionamento adequado de profissionais de enfermagem, preferivelmente, em dois ou mais profissionais, conforme a necessidade do paciente assistido, tornando o banho no leito mais seguro. Os principais riscos para a segurança do paciente durante o banho no leito estão relacionados aos riscos de infecção, de quedas do leito e de deslocamentos de dispositivos (Moller \& Magalhaes, 2015).

Pesquisa multicêntrica realizada na Europa em 24 UTIs em que avaliou as principais repercussões durante o banho no leito na UTI, obteve como resultados mais comuns: dessaturação (5,5\%), hipotensão arterial (4,5\%), agitação (4,2\%) e dor aguda (3,6\%) (Decormeille et al., 2020).

Em estudo observacional, ao relacionar os cuidados de enfermagem antes, durante e após o banho no leito, verificou que ocorrem falhas importantes nos cuidados pertinentes para a execução do banho no leito na UTI. Dentro os cuidados observados, em $86,7 \%$ dos procedimentos observados o paciente foi avaliado quanto a condição de banho, e tal avaliação foi realizada pela equipe técnica, sem participação do enfermeiro. A aspiração de vias aéreas superiores foi realizada quando necessário em $57 \%$ dos casos, a higienização das mãos em apenas 16,7\%, a execução do banho por apenas um profissional em paciente sob ventilação mecânica ocorreu em 23,8\%, higiene oral realizada em apenas 46,7\% dos pacientes observados. Portanto, destacamos a importância da reaproximação do enfermeiro junto a equipe antes, durante e após este cuidado, com objetivo de uma prática de enfermagem guiada por raciocínio clínico e segurança (BASTOS, et al., 2019).

Diante dos principais achados podemos elencar alguns diagnósticos de enfermagem segundo NANDA-I (2018-2020), 
como "padrão respiratório ineficaz caracterizado por taquipneia relacionada ao banho no leito", devido ao aumento da FR. Em relação a queda na temperatura axilar no final do banho podemos associar ao "risco de termorregulação ineficaz relacionada a atividade vigorosa, aumento na demanda de oxigênio e sedação". Relacionada as alterações de pressão arterial podemos associar ao "risco de pressão arterial instável relacionada as alterações de posicionamento do paciente durante o banho no leito".

As limitações apresentadas por esta revisão foram relacionadas a quantidade de artigos selecionados e a falta de investigação quanto as repercussões clínicas geradas pelas alterações hemodinâmicas identificadas.

\section{Conclusão}

Evidenciou-se a necessidade de realizar investigações mais minuciosas para melhor esclarecimento dos impactos do banho no leito ao paciente crítico, para uma assistência de enfermagem com embasamento em evidências cientificas e segura.

No entanto, já podemos observar que apesar de um procedimento rotineiro, e por vez banalizado, o banho no leito do paciente crítico apresenta alterações hemodinâmica importantes e que devem ser consideradas na tomada de decisão dos cuidados de enfermagem. Assim como, a necessidade do profissional enfermeiro agregar conhecimento e avaliação do paciente beira leito antes, durante e após a realização do cuidado do banho no leito. Desta forma, levando a uma assistência de enfermagem mais segura e decisões pautadas em evidências cientificas.

Os estudos selecionados apresentaram grupos de pacientes distintos analisados no contexto do paciente crítico, o que dificulta a generalização dos dados observados. E reforça a necessidade de novas evidências cientificas que identifiquem as repercussões clínicas causadas por tais variações hemodinâmicas, e que norteiem uma assistência de enfermagem segura e individualizada através da avaliação do paciente e de um plano de cuidado para realização da higiene corporal.

\section{Referências}

Backes, M. T. S., Erdmann, A. L. \& Buscher, A. (2015). O ambiente vivo, dinâmico e complexo de cuidados em Unidade de Terapia Intensiva. Rev. LatinoAm. Enfermagem. https://www.revistas.usp.br/rlae/article/view/102730/100978.

Bastos, S. R. B., Gonçalves, F. A. F., Bueno, B. R. M., Silva, G. S., Ribeiro, K. R. A., \& Brasil, V. V. (2019). Bed-Bath: The Care-Omitting Behavior of the Nursing Team. Rev Fund Care Online.http://www.seer.unirio.br/index.php/cuidadofundamental/article/view/6634

Brasil. Ministério da Saúde (2010). RDC $N^{o}$ 7, DE 24 DE FEVEREIRO DE 2010. Agência Nacional de Vigilância Sanitária. 2010. https://bvsms.saude.gov.br/bvs/saudelegis/anvisa/2010/res0007_24_02_2010.html

Costa, G. S., Souza, C. C., Diaz, F. B. B. S., \& Toledo, L. V. (2018). Banho no leito em cuidados críticos: uma revisão integrativa. Rev baiana de enferm. https://periodicos.ufba.br/index.php/enfermagem/article/view/20483.

Decormeille, G., Maouchi, V. M., Mercier G., Debock, S., Lebrun, C., Rouhier, M., \& Lascarrou, J. B (2020). Adverse Events in Intensive Care and Continuing Care Units During Bed-Bath Procedures: The Prospective Observational NURSIng during critical carE (NURSIE) Study. Critical Care Medicine. https://www.srlf.org/files/medias/documents/NURSIE.pdf.

Diagnósticos de enfermagem da NANDA-I: definições e classificação 2018-2020 (2018); tradução: Regina Machado Garcez; revisão técnica: Alba Lucia Bottura Leite de Barros... [et al.].

Flores, G. P. (2016). Critérios para banho no leito em unidade de terapia intensiva adulto: construção de um protocolo assistencial. Dissertação. Universidade do vale do rio dos sinos.

Galvão, C. M., Mendes, K. D. S. \& Silveira, R. C. C. P. (2010). Revisão integrativa: método de revisão para sintetizar as evidências disponíveis na literatura. São Paulo: Iátria.

Galvão, C. M. (2006). Níveis de evidência. Acta Paul Enferm. https://www.scielo.br/j/ape/a/JXrfXqCfD4vPztQFQBr kB7g/?format=pdf\&lang=pt

Madrid, S. Q., Lopez, C. C., Otálvaro, A. F. T. \& Padilla, L. M. R. (2012) Alteraciones hemodinámicas del paciente crítico cardiovascular durante la realización del baño diário. MEDICINA U.P.B. https://revistas.upb.edu.co/index.php/medicina/article/view/1741

Marins, I. F. \& Cruz, I. C. F. (2016). A segurança do paciente na higienização corporal em pacientes de CTI: revisão sistematizada da literatura para um protocolo clínico. Journal of Specialized Nursing Care. http://www.jsncare.uff.br/index.php/jsncare/rt/printerFriendly/2808/682

Moller, G. \& Magalhaes, A. M. M. (2015) Banho no leito: carga de trabalho da equipe de enfermagem e segurança do paciente. Texto Contexto Enferm. https://www.scielo.br/j/tce/a/LYZmW3VFVLsqfgxmpr46WWt/?format=pdf\&lang=pt. 
Research, Society and Development, v. 10, n. 14, e66101421652, 2021

(CC BY 4.0) | ISSN 2525-3409 | DOI: http://dx.doi.org/10.33448/rsd-v10i14.21652

Potter, P. A. \& Perry, A. G. (2013). Fundamentos de enfermagem. Elsevier.

Ramos, J. F. R., Consuegra, R. V. G., \& Urrego, K. A. G. (2016). Modelo de adaptación de Roy en el baño en cama. Av Enferm. https://docs.bvsalud.org/bibli oref/2 020/09/950663/modelo-de-adaptacion-de-roy-en-el-bano-en-cama.pdf

Reis, F. F., Silva, L. S., Sptiz, V. M., Silva, M. E. S., Oliveira, A. P., \& Lima, D. V. M. (2021). Padrão oxihemodinâmico não-invasivo durante o banho no leito de pacientes com infarto agudo do miocárdio: ensaio clínico. Research, Society and Development. https://rsdjournal.org/index.php/rsd/article/view/18237

Toledo, L. V., Salgado, P. O., Souza, C. C., Brinati, L. M., Januário, C. F., \& Ercole. F. F. (2020). Efeitos do banho no leito a seco e tradicional sobre parâmetros respiratórios: estudo piloto randomizado.Rev. Latino-Am. Enfermagem. https://www.scielo.br/j/rlae/a/G4mNDsGp kvv5zGbKBMmDYtf/?format=pdf\&lang=pt.

Toledo, L. V., Santos, B. X., Salgado, P. O., Souza, L. M., Brinati. L. M., Januário, C. F., \& Ercole, F. F. (2021). Alterações na temperatura corporal de pacientes críticos submetidos ao banho no leito: ensaio clínico crossover. Rev Bras Enferm. https://www.scielo.br/ $\mathrm{j} /$ reben/a/JzznTbdJnGXjWKYKQBfKPsb/?lang=pt\&format=pdf

Viana, R. A. P. P., Whitaker, I. Y., \& Zanei, S. S. V. (2020). Enfermagem em terapia intensiva: práticas e vivências [Livro].

Viana, R. A. P. P. (2011). Enfermagem em terapia intensiva: práticas baseadas em evidências [Livro].

Santos, C. M. C., Pimenta, C. A. M., \& Nobre, M. R. C. (2007). A estratégia PICO para construção da pergunta de pesquisa e busca de evidências. Rev Latinoam Enfermagem. https://www.scielo.br/j/rlae/a/CfKNnz8mvSqVjZ37Z77pFsy/?lang=pt 